\title{
Collision-Tolerant Transmission with Narrow-Beam Antennas
}

\author{
Hong-Ning DAI ${ }^{1}$, Kam-Wing NG ${ }^{1}$, Min-You WU ${ }^{2}$, Bo LI ${ }^{3}$ \\ ${ }^{1}$ Department of Computer Science and Engineering, The Chinese University of Hong Kong, Hong Kong SAR \\ ${ }^{2}$ Computer Science and Engineering Department, Shanghai Jiao Tong University, Shanghai, P.R.China \\ ${ }^{3}$ School of Electronic and Information Engineering, South China University of Technology, Guangzhou, P.R.China \\ Email: \{hndai,kwng\}@cse.cuhk.edu.hk,wu-my@sjtu.edu.cn, bill.leebo@gmail.com \\ Received April 29, 2008; revised September 1, 2008; accepted September 23, 2008.
}

\begin{abstract}
The application of directional antennas in wireless ad hoc networks brings numerous benefits, such as increased spatial reuse and mitigated interference. Most MAC protocols with directional antennas are based on the RTS/CTS mechanism which works well in wireless ad hoc networks using omni-directional antennas. However, RTS/CTS frames cannot mitigate the interference completely. Besides, they also contribute a lot to the performance overhead. This paper studies the problem from a new perspective. We have found that the transmission success probability under directional transmission and directional reception is quite high when the antenna beamwidth is quite narrow. Motivated by the analytical results, we design a lightweight MAC protocol without RTS/CTS frames. The evaluation results demonstrate that this new protocol performs better than MAC protocols based on the RTS/CTS mechanism. The results also show that a collision-tolerant transmission is feasible under the narrow beam configuration.
\end{abstract}

Keywords: Wireless Networks, Directional Antennas, Medium Access Control

\section{Introduction}

The application of directional antennas to wireless ad hoc networks has received enormous interest in recent years. Directional antennas can greatly improve network performance by increasing network connectivity, expanding transmission range, enhancing spatial reuse and reducing interference. Recent studies such as [1-10] focus on designing new MAC layer protocols to improve network performance.

Most of these MAC schemes with directional antennas are based on a four-way handshaking scheme, known as request-to-send/clear-to-send (RTS/CTS). The RTS/CTS mechanism has been proposed to resolve the hidden terminal problem in wireless networks using omni-directional antennas which can broadcast RTS/CTS frames to inform neighboring nodes of the oncoming transmission. Those nodes that have received the RTS/CTS frames can defer their transmission to avoid collisions. However, using RTS/CTS cannot eliminate hidden terminals completely even in wireless networks with omni-directional antennas
[11]. Furthermore, Choudhury et al. [5] have found that using directional antennas causes new interference such as new hidden terminals and the deafness problem, which cannot be solved by using the RTS/CTS mechanism. Essentially, directional antennas can radiate or receive signals more effectively in one direction, which can cause much less interference than omni-directional antennas. So, does the RTS/CTS mechanism still work well with directional antennas?

Many novel mechanisms have been proposed to eliminate the new hidden terminal problem and the deafness problem, which are caused by directional antennas. Although Korakis et al. [3] propose a CircularDMAC scheme to combat the new hidden terminal problem and the deafness problem, transmitting multiple RTS/CTS frames for each data transmission severely degrades the performance. Other schemes, such as Tonebased DMAC [7] and BTDMAC [12] can alleviate the impacts of the hidden terminal and deafness problems by sending tones over another channel or over the data channel after data transmission. However, these bulky and complicated schemes also bring additional cost and 
performance penalty.

How to use directional antennas in wireless networks more effectively? We address this problem from another viewpoint. When the beamwidth of a directional antenna is lessened (a narrower beamwidth), the interference caused by the antenna will also be reduced. We have found that when the beamwidth is quite narrow and the network is not so dense, the collision probability is quite low. It is the purpose of this paper to study the performance of wireless networks using narrow-beam antennas. In particular, we are interested in the following problems:

- What will happen when the beamwidth of the directional antennas is lessened? What is the impact of other factors on the success transmission probability, such as the node density?

- How effective is the RTS/CTS mechanism in wireless networks using directional antennas? If RTS/CTS is turned off, will the network throughput degrade significantly?

In the next section, we briefly survey the related work in the literature. Section 3 describes the models used in this paper and analyzes the success transmission probability for directional transmission and directional reception. In Section 4, we present a lightweight MAC protocol without the RTS/CTS mechanism and compare its performance with a representative MAC protocol using the RTS/CTS mechanism. Section 5 offers some implications of our results. Finally, we summarize our paper in Section 6.

\section{Related Work}

Many studies [1-10] focus on designing new MAC protocols with directional antennas. Most of them are based on the IEEE 802.11MAC [13], which typically uses RTS/CTS to reduce interference in wireless networks. Although the RTS/CTS mechanism works well in wireless networks equipped with omni-directional antennas, it cannot mitigate interference completely [11]. Besides, using RTS/CTS in wireless networks with directional antennas is not as effective as we expected. For example, ref. [5] shows that RTS/CTS cannot completely mitigate new interfering nodes caused by directional antennas.

To address the new hidden terminal problem and the deafness problem, many researchers propose more complex schemes, such as Circular-DMAC [3], Tonebased DMAC [7] and BT-DMAC [12]. Although they can mitigate the impacts of hidden terminals and deafness, they also bring additional cost on network performance. For example, Circular-DMAC needs a sender to transmit multiple RTS frames before each data transmission, which greatly degrades the network performance. Tonebased DMAC and BT-DMAC also need to send out-ofband tone signals to reduce interference.

Other studies [14-17] concentrate on capacity analysis and performance evaluation on wireless ad hoc networks using directional antennas. Yi et al. [15] have found that using directional antenna in arbitrary networks achieves a capacity gain of $2 \pi / \sqrt{\alpha \beta}$ when both transmission and reception are directional. Here, $\alpha$ and $\beta$ are transmitter and receiver antenna beamwidths, respectively. Under random networks, the throughput improvement factor is $4 \pi^{2} /(\alpha \beta)$ for directional transmission directional reception. Ref. [14] studies the asymptotic bounds on the amount of capacity gains that directional antennas can acquire. Wang et al. [16] model and analyze multiple directional transmission and reception modes coupled with omni-directional or directional receptions. Carvalho and Garcia-Luna-Aceves [17] propose a realistic analytical model which considers the binary exponential back-off operation of IEEE 802.11.

In this paper, we try to find the relationship between the interference, the beamwidth of directional antennas and the density of nodes.

\section{Analytical Models}

In this section, we analyze the successful transmission probability with directional antennas. The successful transmission probability does not only depend on the activity of the interfering nodes but also on the transmission/reception mode of directional antennas. First, we present the antenna model in Section 3.1. Section 3.2 discusses the interference range for directional transmission. Finally, we analyze the successful transmission probability under the directional transmission and directional reception mode.

\subsection{Antenna Model}

The radiation pattern of a direction antenna is often depicted as the gain values in each direction in space. We can project the radiation pattern of an antenna to an azimuthal or elevation plane. The projection of the pattern typically has a main lobe (beam) of the peak gain and side-/back-lobes of smaller gains.

Since modeling a real antenna with precise values for main and side-/back-lobes is difficult, we use an approximate

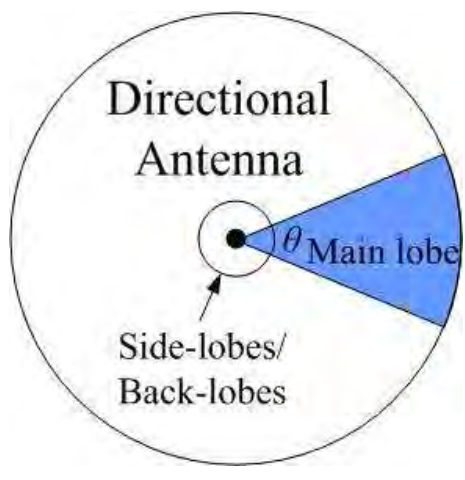

Figure 1. The antenna model. 
antenna pattern [18]. In an azimuthal plane, the main lobe of antenna can be depicted as a sector with angle $\theta$, which is denoted as the main beamwidth of the antenna. The side-/back-lobes are aggregated to a circle, as shown in Figure 1. The narrower the main beamwidth of the antenna is, the smaller the side-/back-lobes are. Take the above antenna model as the example. The gain of the main beam is more than 100 times of the gain of sidelobes when the main beamwidth is less than $40^{\circ}$ [18]. Thus, the side-/back-lobes can be ignored when the main beam is quite narrow.

Our proposed model assumes that a directional antenna gain $G^{d}$ is within a specific angle $\theta$, where $\theta$ is the beamwidth of the antenna. The gain outside the beamwidth is assumed to be zero. At any time, the antenna beam can only be pointed to a certain direction, as shown in Figure 1, in which the antenna is pointing to the right. Thus, the probability that the beam is switched to cover each direction is $\theta /(2 \pi)$. The antenna gain pattern is given by:

$$
g(\theta)=\left\{\begin{array}{cr}
G^{d} & \text { if angle within } \theta \\
0 & \text { otherwise }
\end{array}\right.
$$

\subsection{Interference Range}

Compared with omni-directional antennas, directional antennas have different transmission properties. Directional antennas can radiate or receive ratio signals more effectively in a certain direction than other directions. Thus, directional antennas have different transmission range and interference region, compared with omni-directional antennas. In this subsection, we investigate the interference region of directional antennas and the relationship between the transmission range and the interference range.

When a signal is propagated from the transmitter to the receiver, whether it is correctly accepted by the receiver is mainly determined by the receiving power of the signal at the receiver end. In open space, if the transmitting power is fixed, the receiving power is mostly decided by the path loss along the distance between the transmitter and the receiver. Under this condition, multipath and shadowing effects can be ignored since they are so trivial compared with the large path loss. Therefore, in this paper, we assume that the signal propagation follows the two-way ground model which is typically used in open space.

According to [19], under the assumption of the twoway ground model, the receiving power of a signal at the receiver can be calculated by the following equation.

$$
P_{r}(d)=P_{t} G_{t} G_{r} \frac{h_{t}^{2} h_{r}^{2}}{d^{4}}
$$

where $P_{r}(d)$ is the receiving power at the receiver which is far from the transmitter with the distance $d, P_{t}$ is the

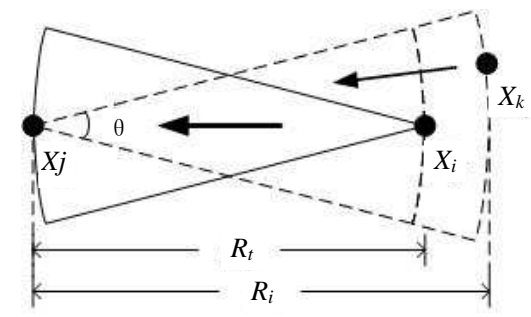

Figure 2. The interference model.

transmitter power, $G_{t}$ and $G_{r}$ are the transmitter antenna gain and the receiver antenna gain, respectively, and $h_{t}$ and $h_{r}$ are the antenna height of the transmitter and the antenna height of the receiver, respectively.

Consider a large-scale wireless ad hoc network with $n$ static nodes. Without loss of generality, the distribution of the nodes follows a Poisson distribution with a parameter $\rho$ over the 2-D plane. The probability $p(i, S)$ of finding $i$ nodes in an area of $S$ is given by:

$$
p(i, S)=\frac{(\rho S)^{i}}{i !} e^{-\rho S}
$$

We also assume that every node has an identical antenna and transmits with a fixed power. Thus, each node has the same transmitting range $R_{t}$ and the same interference range $R_{i}$. In the scenario shown in Figure 2, suppose that node $X_{i}$ transmits to node $X_{j}$ over a channel. The receiver $X_{j}$ locates exactly within the transmitting range $R_{t}$ of the transmitter $X_{i}$.

The successful reception of the signal is mainly decided by the signal-to-interference-plus-noise ratio (SINR), which is often required to be greater than a threshold. When their transmission is on-going, an interfering node $X_{k}$ at the interference range $R_{i}$ away from the receiver starts the transmission toward the receiver at the same time. So, it will have an interfering signal with the strength $P_{i}$ at the receiver $X_{j}$. Since the thermal noise is negligible compared with interference signals, similar to [11], we do not count it in our model as well. Thus, we have $\operatorname{SINR}=\frac{P_{r}}{P_{t}}=\frac{R_{i}^{4}}{R_{t}^{4}} \geq \sigma$, where $\sigma$ is the SINR threshold. In practice, $\sigma$ is usually set to 10 . So, we get the interference range $R_{i}=\sqrt[4]{\sigma} R_{t}$.

\subsection{Directional Transmission and Directional Reception}

A directional antenna has two modes: an omnidirectional mode with a gain $G^{o}$ and a directional mode with a gain $G^{d}$. Since antennas in the directional mode can radiate or receive radio waves more effectively in 
some directions than in others, the directional gain $G^{d}$ is generally greater than the omni-directional gain $G^{o}$. The transmitter or the receiver equipped with a directional antenna can choose any one of the two modes to transmit or receive frames. Hence, there are four combinations for the transmission and reception modes of directional antennas: 1) Omni-directional Transmission and Omnidirectional Reception (OTOR); 2) Directional Transmission and Omni-directional Reception (DTOR); 3) Omni-directional Transmission and Directional Reception (OTDR); 4) Directional Transmission and Directional Reception (DTDR).

According to Equation (1), the larger the antenna gains both the transmitter and the receiver have, the higher the receiving power the receiver has. Besides, the transmission range between the transmitter and the receiver will be extended if the antenna gains of them are increased. Thus, when both the receiver and the transmitter use the directional mode, the communication range between them is maximized. On the other hand, the receiver is only susceptible to the interfering signals from its receiving direction when it is using the directional mode. So, DTDR also has the smallest interference area compared with the other three modes. Hence, DTDR is a preferred method to utilize directional antennas. In this paper we only discuss the transmission under the DTDR mode.

Let us consider the scenario shown in Figure 2. When node $X_{i}$ begins to transmit with node $X_{j}$, this packet is successfully received by node $X_{j}$ if no node within the sector region covered by $X_{j}$ 's antenna beam transmits toward $X_{j}$. First, we need to calculate the probability that no node can interfere with node $X_{j}$. Since the placement of nodes follows the 2-D Poisson distribution with the density $\rho$, there are $\rho \pi R_{i}^{2} \cdot \frac{\theta}{2 \pi}$ nodes within the sector region covered by $X_{j}$ 's antenna beam. The area of this region is denoted by $S$. Among these nodes, the interfering node $X_{k}$ can cause interference with node $X_{j}$ only when it has a frame to send and its antenna beam is pointed to node $X_{j}$. We assume that a node begins to transmit with a probability $p$.Then, the probability that node $X_{k}$ can interfere with node $X_{j}$ is $p \cdot \frac{\theta}{2 \pi}$. There, the probability $P$ that no nodes within region can cause collisions with node $X_{j}$ is given by:

$$
\begin{aligned}
& P=\sum_{i=0}^{\infty}\left(1-p \frac{\theta}{2 \pi}\right)^{i} \cdot \frac{(\rho S)^{i}}{i !} e^{-\rho S} \\
& =e^{-p \frac{\theta}{2 \pi} \rho S}=e^{-p\left(\frac{\theta}{2 \pi}\right)^{2} \rho \pi R_{i}^{2}}
\end{aligned}
$$

To simplify the calculation, we use $N=\rho \pi R_{t}^{2}$, which denotes the average number of nodes within a node's trans-

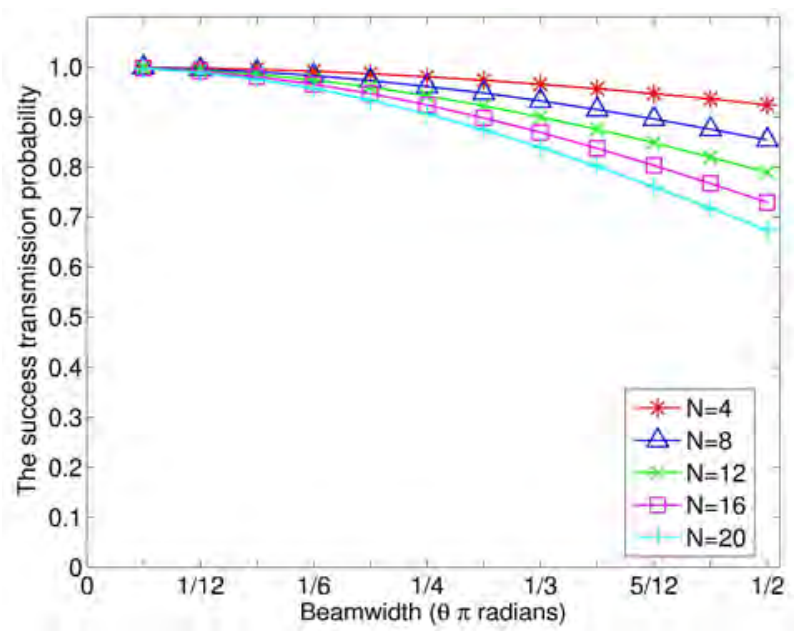

Figure 3. The probability of a successful transmission.

mission range. Since $R_{i}=\sqrt[4]{\sigma} R_{t}$, we have $\rho \pi R_{i}^{2}=\sqrt{\sigma} N$. Replacing the corresponding part in Equation (3), we have:

$$
P=e^{-p\left(\frac{\theta}{2 \pi}\right)^{2} \sqrt{\sigma} N}
$$

When $p=0.1$ and $\sigma=10$, we set different $N=4,8,12,16,20$ respectively and then we get the results in Figure 3. Figure 3 shows that the successful transmission probability is high when the beamwidth is narrow. For example, when $\theta$ is less than $\frac{\pi}{6}$, the success probability is always above $95 \%$. One possible reason is that using directional mode at the receiver end can greatly reduce the collision probability.

Results under a narrow beamwidth $\left(\theta \leq \frac{\pi}{12}\right)$ are also tabulated in Table 1, which shows that the transmission under DTDR is less vulnerable to interference when the beamwidth is quite narrow.

The analytical results under DTDR show that the successful transmission probability is quite high when the beamwidth is lessened enough. For example, when $\theta \leq \frac{\pi}{12}$ (i.e., $15^{\circ}$ ), the success probability is always above $98 \%$. A beamwidth of $15^{\circ}$ is a feasible angle for most directional antennas. Thus, intuitively, there is an

Table 1. The probability of a successful transmission under the very narrow beam.

\begin{tabular}{lclll}
\hline & $\theta=\frac{\pi}{48}$ & $\theta=\frac{\pi}{36}$ & $\theta=\frac{\pi}{24}$ & $\theta=\frac{\pi}{12}$ \\
\hline$N=4$ & 0.9999 & 0.9998 & 0.9995 & 0.9978 \\
$N=8$ & 0.9997 & 0.9995 & 0.9989 & 0.9956 \\
$N=12$ & 0.9996 & 0.9993 & 0.9984 & 0.9934 \\
$N=16$ & 0.9995 & 0.999 & 0.9978 & 0.9913 \\
$N=20$ & 0.9993 & 0.9988 & 0.9973 & 0.9891 \\
\hline
\end{tabular}


interesting question: can the transmission continue even if there exist few collisions? In other words, when the beamwidth of antennas is narrow enough and the collision probability is quite low, can the transmission be collision-tolerated?

\section{Lightweight MAC Protocol}

In this section, we propose a lightweight MAC scheme denoted as Basic Directional Transmission and Directional Reception (B-DTDR), which turns off the control frames of request to send (RTS) and clear to send (CTS). It has a rival termed RTC/CTS Directional Transmission and Directional Reception (RTS-DTDR). Then, we compare the performance of B-DTDR with that of RTS-DTDR and discuss the implications from this lightweight scheme.

\subsection{Quick Review of RTS/CTS Mechanisms with Directional Antennas}

B-DTDR scheme keeps the basic collision avoidance features, such as the exponential backoff and the control frame of acknowledge (ACK). Thus, if there is a collision with data packets, those data packets need to be retransmitted. In IEEE 802.11 distributed coordination function (DCF) [13], an exponential backoff scheme can be used to avoid further collisions of data packets. At each packet transmission, the backoff time is uniformly chosen in a range of $(o, w)$ where $w$ is called the contention window. At the first transmission, $w$ is set to be the minimum value of $W_{\min }$. After each unsuccessful transmission (no ACK received), the size of $w$ is doubled until it reaches the maximum value of $W_{\max }$. This mechanism can effectively reduce the collision probability.

Most of current directional MAC schemes are using another four-way handshaking technique which turns on RTS/CTS frames. In stead of sending a data packet, a transmitter sends a short control frame called request to send (RTS). After the reception of RTS, the receiver responds the transmitter with the frame called clear to send (CTS). After shaking hands of RTS and CTS, the data transmission begins. This mechanism is useful to reduce the hidden nodes in wireless networks using omni-directional antennas. However, it cannot mitigate the hidden terminal problem and the deafness problem with directional antennas [5]. In this paper, we consider a general MAC scheme (RTSDTDR) which can be used to represent the current directional MAC schemes since it keeps has the main features of them. In both B-DTDR and RTS-DTDR, RTS, CTS, data packets and ACK are transmitted directionally.

One of the difficult problems with B-DTDR and RTSDTDR is to find the location of a node's neighbors, or neighbor discovery. This problem can be solved by using DOA caching [4] or similar mechanisms. A specific problem with B-DTDR is how to help a receiver to know that a transmitter is trying to send a frame to it. Zhang [10] proposes a scheduling mechanism to address this problem. In this paper, we assume that both B-DTDR and RTS-DTDR can solve the neighbor discovery problem.

On the other hand, when a node receives any frames (RTS, CTS and data frames), it will record the corresponding information into its DNAV (Directional Network Allocation Vector), which is a directional version of NAV of IEEE 802.11, proposed in [4,5]. DNAV excludes the directions and sets the corresponding durations, toward which the node is not allowed to initiate a transmission to avoid collisions with data or control frames. When a node receives a frame and the frame is for this node, it beamforms toward the transmitter (switch to directional mode) and replies the frame with a CTS (or ACK) frame. If the frames are not for itself, it will update the sender's information and set the corresponding DNAVs. DNAVs are used in both BDTDR and RTS-DTDR.

\subsection{Performance Model}

In this paper, we adopt a discrete Markov chain model used in $[16,20]$ to evaluate the saturation throughput and the overhead of wireless networks (as shown in Figure 4). We extend the model to support directional antennas. Range extension and overhead calculation are also considered in our model. We also adopt the assumption that each node operate in time-slotted mode, with a time slot $\tau$. If the time slot $\tau$ is very small, the performance of the time-slotted protocol is very close to that one of the asynchronous version of the protocol $[16,20]$. The period of time during which RTS, CTS, data and ACK frames are transmitted can be depicted as multiples of $\tau$, i.e., $t_{r t s}, t_{c t s}, t_{\text {data }}$ and $t_{\text {ack }}$, respectively.

The throughput is calculated by the proportion of time that a node spends transmitting data packets successfully on the average. Let $P(S), P(I)$ and $P(C)$ denote the steady-state probability of SUCCESS, IDLENESS and COLLISION, respectively. From the Markov chain model

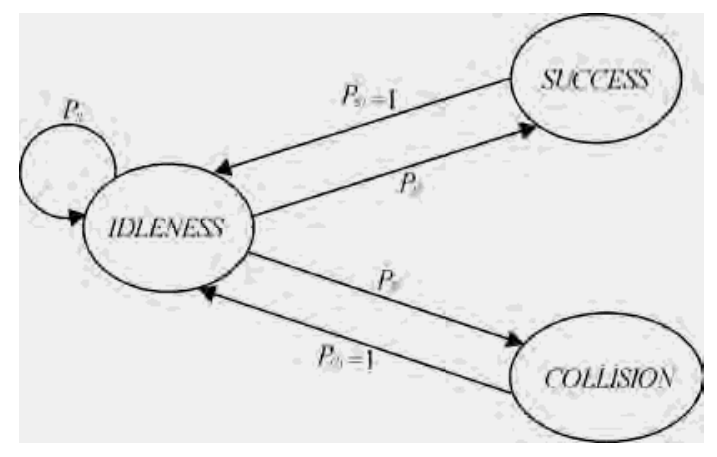

Figure 4. The Markov chain model for a node. 
shown in Figure 4, we have the following equation to calculate the throughput.

$$
\text { Throughput }=\frac{P(S) \cdot t_{\text {data }}}{P(C) T_{C}+P(S) T_{S}+P(I) T_{I}}
$$

where $T_{C}, T_{S}$ and $T_{I}$ are the duration of COLLISION, SUCCESS and IDLENESS, respectively.

The duration of time that a node stays in the SUCCESS state, $T_{S}$ or the collision state, $T_{C}$, depend on the mechanisms of different MAC protocols. Thus, the detailed calculation will be given in the following subsections. The duration of a node in IDLENESS state $T_{I}$ is $1 \tau$

Then, we need to calculate the probabilities that the node stays in different states. From Figure 4, the steadystate probability of IDLENESS equals:

$$
P(I)=P(I) \cdot P_{I I}+P(S)+P(C)
$$

Note that $P(S)+P(C)=1-P(I)$, thus,

$$
P(I)=1 /\left(2-P_{I I}\right)
$$

From Figure 4, the steady-state probability of $S U C C E S S$ can be calculated by $P(S)=P(I) \cdot P_{I S}$. Before deriving the transition probability $P_{I S}$ from IDLENESS to $S U C C E S S$, we need to calculate $P_{I S}(r)$ that node $X_{i}$ successfully shakes hands with node $X_{j}$ which is a distance $r$ way. The detailed calculation of $P_{I S}(r)$ will be stated as follows.

We also derive the MAC overhead by calculating the portion of time that a node spends transmitting control frames on the average when data packets are successfully transmitted.

$$
\text { Overhead }=\frac{P(S) \cdot t_{c t r l}}{P(C) T_{C}+P(S) T_{S}+P(I) T_{I}}
$$

where $t_{c t r l}$ is depicted as time slots which are used to transmit control frames such as RTS, CTS and ACK.

In the following subsections, we derive the steadystate probabilities, transition probabilities and times spent at different states of the two MAC schemes, respectively.

\subsection{RTS/CTS Based Directional Transmission and Directional Reception (RTS-DTDR)}

In this subsection, we calculate the throughput and the overhead of RTS-DTDR. From the throughput model presented above, we need to calculate the transition probability $P_{I S}$ first. Figure 5 indicates that the nodes within the four regions (named 1, 2, 3, 4) may interfere

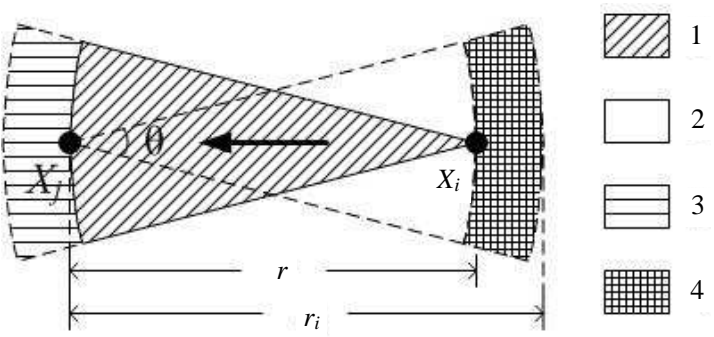

Figure 5. The interference region for DTDR.

with node $X_{i}$ and node $X_{j}$. The transmission range between $X_{i}$ and $X_{j}$ is denoted as $r$. So, the interference range $r_{i}=\sqrt[4]{\sigma} r$, which can be easily derived from the results presented in Section 3.2. Since the number of nodes depends on the area size, we need to calculate the four areas of regions 1, 2, 3 and 4, which are denoted as $S_{1}, S_{2}, S_{3}$ and $S_{4}$, respectively:

$$
\begin{aligned}
& S_{1}=\pi r^{2} \cdot \theta /(2 \pi) \\
& S_{2}=\pi r^{2} \cdot \theta /(2 \pi)-r^{2} \tan (\theta / 2) / 2 \\
& S_{3}=\pi r_{i}^{2} \cdot \theta /(2 \pi)-\pi r^{2} \cdot \theta /(2 \pi) \\
& S_{4}=\pi r_{i}^{2} \cdot \theta /(2 \pi)-\pi r^{2} \cdot \theta /(2 \pi)
\end{aligned}
$$

$P_{I S}(r)$ equals the probability that $X_{i}$ transmits in a given time slot, and $X_{j}$ does not transmit in the same time slot, and none of the nodes within the four regions interferes with the handshake between nodes $X_{i}$ and $X_{j}$. Therefore, we have:

$$
P_{I S}(r)=p(1-p) \cdot P_{1} \cdot P_{2} \cdot P_{3} \cdot P_{4}
$$

Since RTS-DTDR does not prevent interference from neighboring nodes in regions 3 and 4, the handshake might be interrupted at any time. Hence, the COLLISION period $T_{C}$ lasts from

$T_{1}=t_{r t s}+1$ till $T_{2}=t_{r t s}+t_{c t s}+t_{\text {data }}+t_{\text {ack }}+4$, where one propagation delay $1 \tau$ is also considered. $T_{C}$ is the mean value of the truncated geometric distribution. Then, we obtain the following equation to calculate $T_{C}$.

$$
T_{C}=(1-p) /\left(1-p^{T_{2}-T_{1}+1}\right) \sum_{i=0}^{T_{2}-T_{1}} p^{i}\left(T_{1}+i\right)
$$

The probability that no nodes in region 1 interferes with the handshake between nodes $X_{i}$ and $X_{j}$ is equal to the probability that no node in this area transmits as node $X_{i}$ does, which can be depicted as:

$$
\begin{gathered}
P_{1}=\sum_{i=0}^{\infty}(1- \\
\left.=\frac{\theta}{2 \pi}\right)^{i} \cdot \frac{\left(\rho S_{1}\right)^{i}}{i !} e^{-\rho S_{1}} \\
=e^{-p \rho S_{1} \frac{\theta}{2 \pi}}
\end{gathered}
$$


The probability $P_{2}$ is equal to the probability that no node transmits toward node $X_{j}$ within the duration of time $t_{r t s}$ and no node transmits within the slot when node $X_{i}$ begins to transmit toward node $X_{j}$ Thus, we have the following equation to calculate $P_{2}$.

$$
P_{2}=e^{-p \frac{\theta}{2 \pi} \rho S_{2}\left(t_{r s}+1\right)} \cdot e^{-p \frac{\theta}{2 \pi} \rho S_{2}}
$$

$P_{3}$ is equal to the probability that no node can interfere with the reception of CTS and ACK frames of node $X_{i}$. Hence, we have:

$$
P_{3}=e^{-p \frac{\theta}{2 \pi} \rho S_{3}\left(t_{c s s}+1\right)} \cdot e^{-p \frac{\theta}{2 \pi} \rho S_{3}\left(t_{a c k}+1\right)}
$$

In region 4, there is no interference if no node transmits toward node $X_{j}$ when node $X_{i}$ is sending a data frame. Then, we get:

$$
P_{4}=e^{-p \frac{\theta}{2 \pi} \rho S_{4}} \cdot e^{-p \frac{\theta}{2 \pi} \rho S_{4}\left(t_{\text {data }}+1\right)}
$$

Because each transmitter can choose its receiver with the equal probability and the average number of nodes within a region of radius $r$ is proportional to $r^{2}$, the probability density is the function of distance $r$ between nodes $X_{i}$ and $X_{j}$, i.e., $f(r)=2 r$, where $0<r<R_{t}$. Therefore, $P_{I S}$ is equal to:

$$
\begin{gathered}
P_{I S}=\int_{0}^{R_{t}} P_{I S}(r) f(r) d r \\
=\int_{0}^{R_{t}} p(1-p) \cdot P_{1} \cdot P_{2} \cdot P_{3} \cdot P_{4} \cdot 2 r d r
\end{gathered}
$$

The duration in time slots of a node in the SUCCESS state is

$$
\begin{gathered}
T_{S}=\left(t_{r t s}+1\right)+\left(t_{c t s}+1\right)+\left(t_{\text {data }}+1\right)+\left(t_{a c k}+1\right) \\
=t_{r t s}+t_{c t s}+t_{\text {data }}+t_{\text {ack }}+4
\end{gathered}
$$

where $t_{r t s}, t_{c t s}, t_{d a t a}$ and $t_{a c k}$ are the duration times of transmitting RTS, CTS, data and ACK frames, respectively.

After the corresponding parts are replaced in Equation (5), the throughput of RTS-DTDR is obtained. Following the similar process, we can calculate the overhead of RTS-DTDR from Equation (8).

\subsection{Basic Directional Transmission and Directional Reception (B-DTDR)}

Since there is no RTS and CTS frames, B-DTDR has a narrower bound on $T_{C}$ (from $T_{1}=1 \tau$ to $\left.T_{2}=t_{\text {data }}+t_{\text {ack }}+2\right)$. Then we can calculate $T_{C}$ by using
Equation (11).

And the success period time is

$$
T_{S}=t_{\text {data }}+t_{\text {ack }}+2
$$

$P_{1}$ keeps the same as RTS-DTDR. $P_{2}$ is equal to the probability that no node transmits toward node $X_{j}$ within $t_{\text {data }}$ period and does not transmit in the slot when node $X_{i}$ begins to transmit with node $X_{j}$, therefore, we have

$$
P_{2}=e^{-p \frac{\theta}{2 \pi} \rho S_{2}\left(t_{\text {data }}+1\right)} \cdot e^{-p \frac{\theta}{2 \pi} \rho S_{2}}
$$

Similarly, we have

$$
\begin{gathered}
P_{3}=e^{-p \frac{\theta}{2 \pi} \rho S_{3}\left(t_{\text {ack }}+1\right)} \\
P_{4}=e^{-p \frac{\theta}{2 \pi} \rho S_{4}} \cdot e^{-p \frac{\theta}{2 \pi} \rho S_{4}\left(t_{\text {data }}+1\right)}
\end{gathered}
$$

Then after replacing the corresponding parts in Equation (5), we get the throughput of B-DTDR. Since $t_{c t r l}=t_{a c k}+2$ in B-DTDR, we can calculate the overhead of B-DTDR from Equation (8).

\subsection{Numerical Results}

We compare the performance of the RTS-DTDR and BDTDR under the different configurations and present the results in Figure 6 and Figure 7.

Figure 6 shows the saturation throughput and overhead of RTS-DTDR and B-DTDR under different node density ( $N=10,20,30,40$, respectively) when the beamwidth is less than $\frac{\pi}{6}$. The results are obtained under a short data length, i.e., $t_{\text {data }}=40 \tau$. With the increased node density, both the throughputs of RTS-DTDR and BDTDR begin to degrade although B-DTDR has a much higher throughput than RTSDTDR protocol. The peak value of B-DTDR is almost $20 \%$ higher than that of RTS-DTDR. One possible reason is that when the beamwidth is quite narrow, the number of the interfering nodes is so small that those nodes cause nearly no collisions. In this situation, RTS/CTS frames are not necessary to be used. On the contrary, they only contribute additional overhead on the throughput.

Then we calculate the throughput and overhead under the long data length setting (i.e., $t_{\text {data }}=120 \tau$ and the results are shown in Figure 7. Similarly, both RTSDTDR and B-DTDR perform well under a narrow beam (e.g., $\frac{\pi}{15}$ ). Under this setting, B-DTDR still has a higher throughput than RTS-DTDR because it gets rid of the bulky RTS/CTS mechanism. However, when the beamwidth is increased further, the collisions caused by 


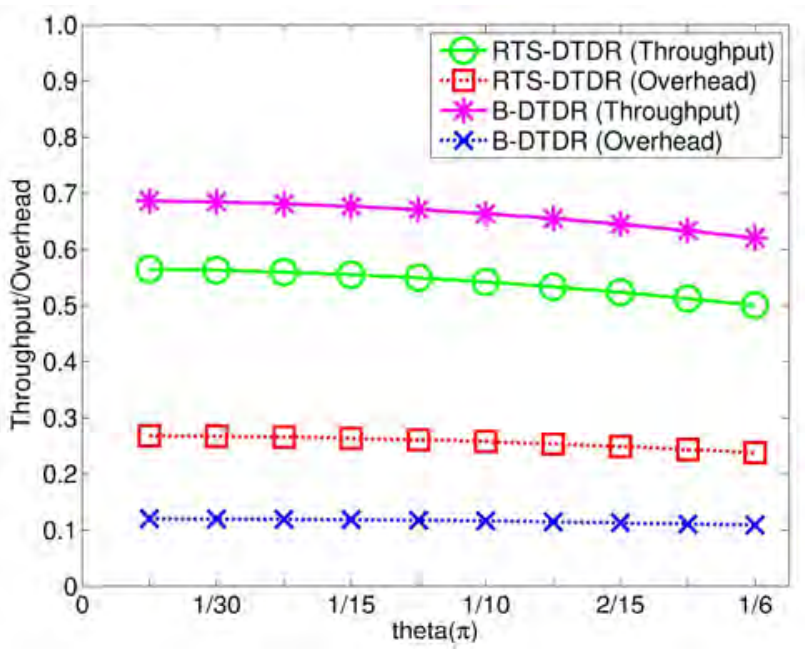

(a) $N=10$

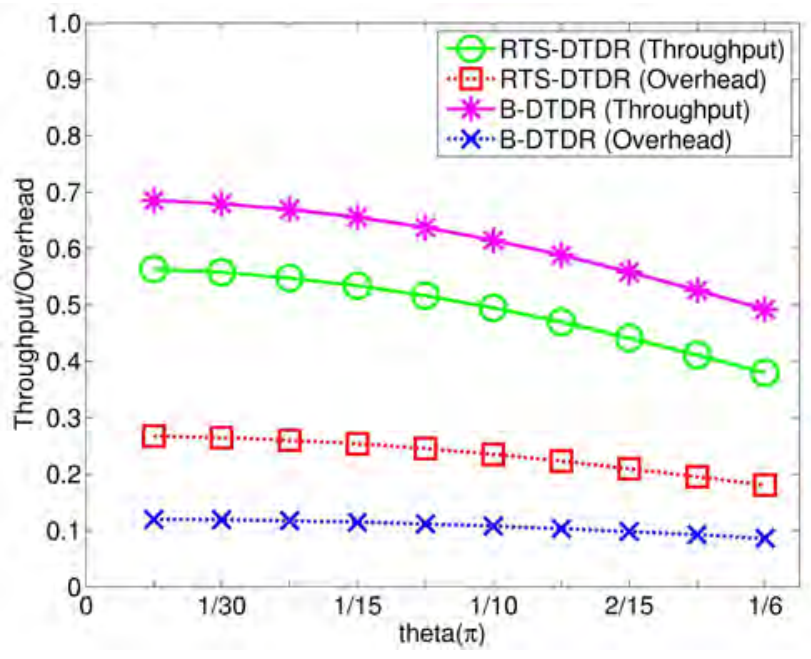

(a) $N=30$

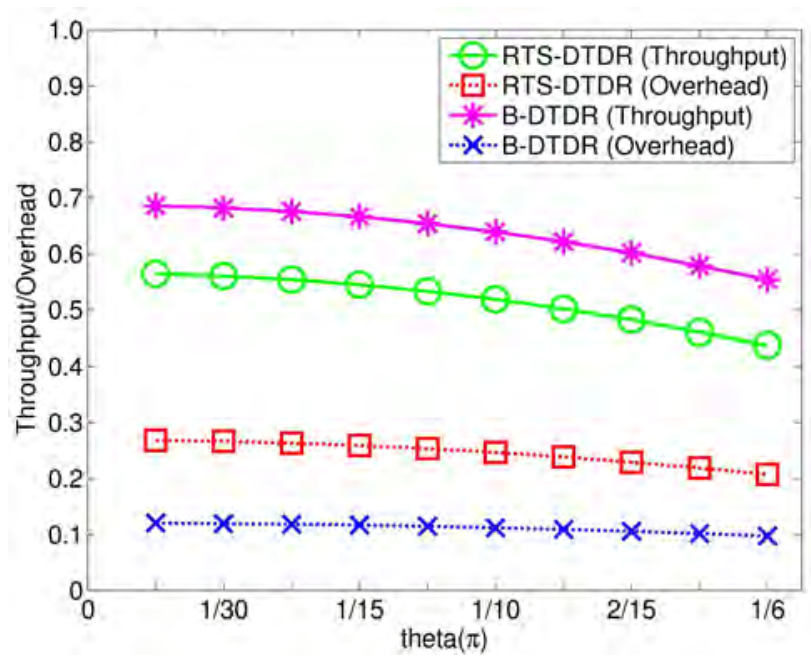

(b) $N=\mathbf{2 0}$

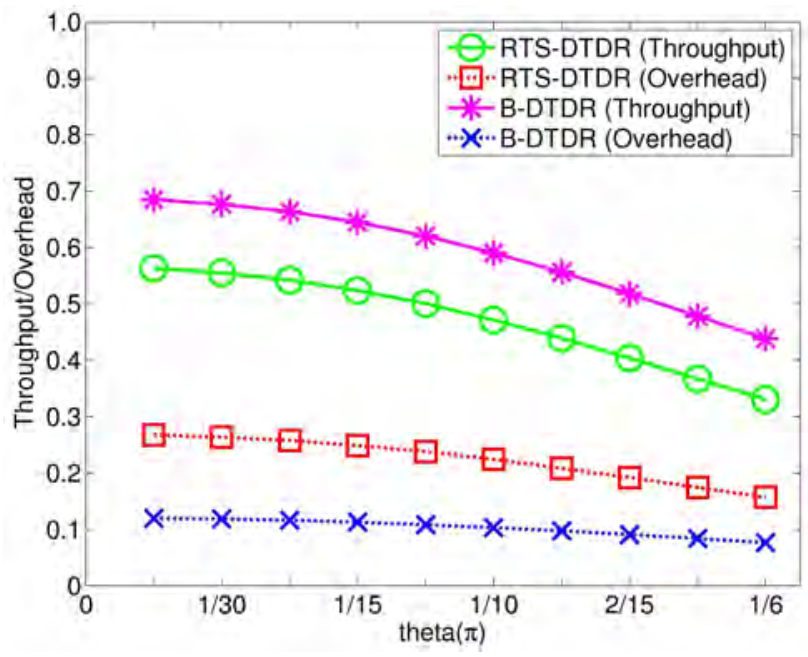

(b) $N=\mathbf{4 0}$

Figure 6. Throughput comparison when $p=0.1, t_{r t s}=t_{c t s}=t_{a c k}=5 \tau, t_{d a t a}=40 \tau$ (short data frame).

interfering nodes become remarkable, both the throughput of RTS-DTDR and B-DTDR degrades.

\section{Discussions}

The results in Figure 6 and Figure 7 show that, when the beamwidth is decreased, a higher network throughput can be obtained. The capacity analysis in [15] also proves that the capacity grows with the lessened beamwidth. However, the capacity will not grow arbitrarily high when the beamwidth decreases further and even approaches to zero. Yi et al. [15] have also observed that when the beamwidth is too small, the interference has been fully reduced and there is no further improvement by decreasing the beamwidth of the antennas.

Actually, when the beamwidth is narrow enough (more specifically, less than a certain angle) a trans-mission can yield a high success probability. As shown in Section 3.3, if the beamwidth is less than $\frac{\pi}{12}$ (i.e., $15^{\circ}$ ) and both directional antennas are used at the transmitter and the receiver, then the probability of a successful transmission is greater than $99 \%$. The transmission under this situation can be regarded as a collision-tolerant transmission (the collision probability is quite small). Hence, DTDR should be the best way to use directional antennas. Meanwhile, the angle $15^{\circ}$ is feasible in most intelligent directional antennas. Under this condition, the complicated collision avoidance mechanisms, such as RTS/CTS, are not necessary to be used because they only contribute excessive overhead on the performance. At that time, using some simple collision avoidance mechanisms, such as the exponential back-off, might be enough to reduce the interference. 


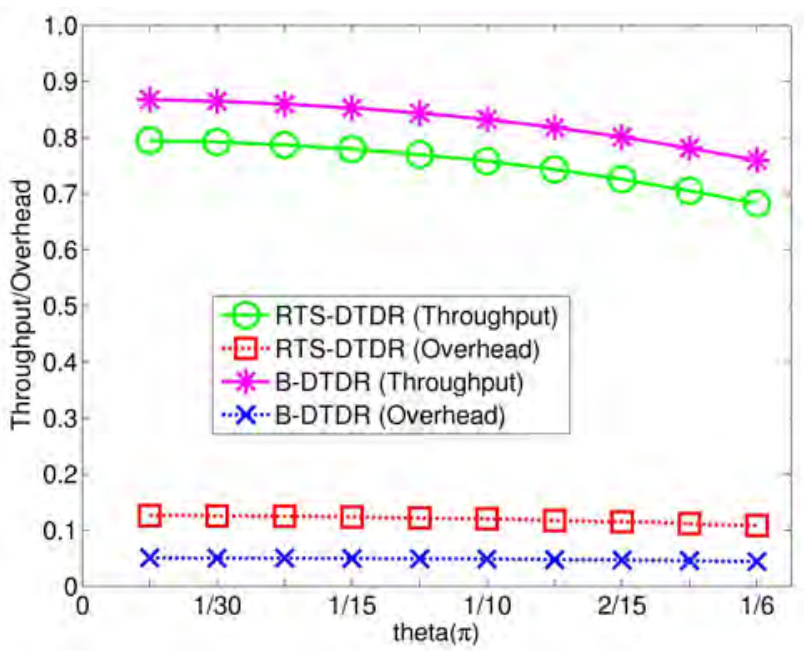

(a) $N=10$

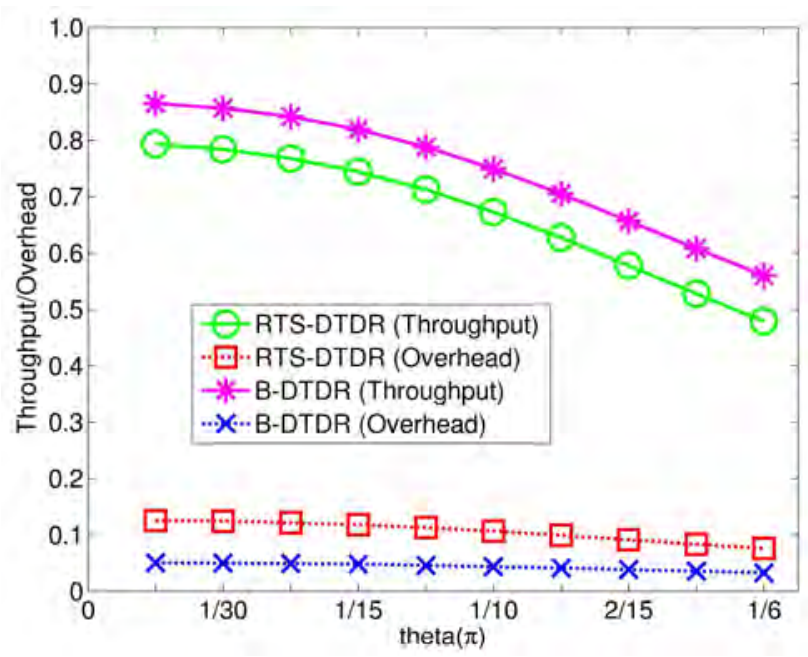

(a) $N=\mathbf{3 0}$

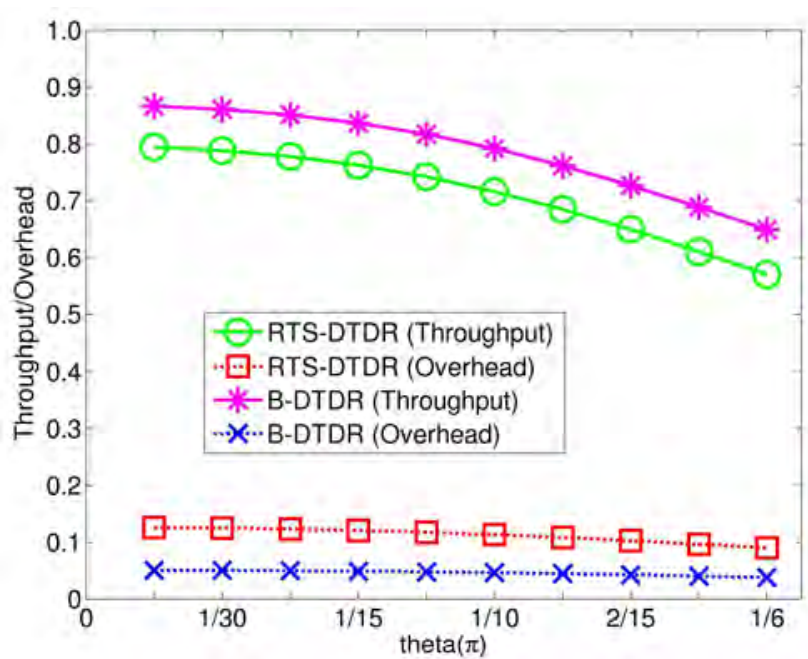

(b) $N=20$

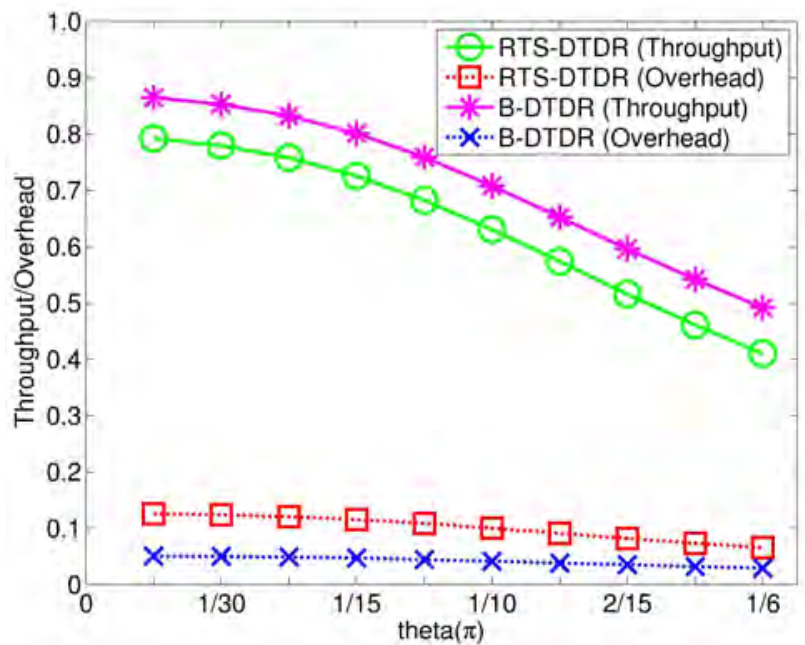

(b) $N=\mathbf{4 0}$

Figure 7. Throughput comparison when $p=0.1, t_{r t s}=t_{c t s}=t_{a c k}=5 \tau, t_{\text {data }}=120 \tau$ (long data frame).

This collision-tolerant transmission gives us some important implications on MAC design. Directional antennas have different properties, e.g., higher spatial reuse and the smaller interfering region. Although RTS/CTS schemes work well in wireless networks using omni-directional antennas, they cannot mitigate interference caused by directional antennas completely [5]. Thus, the MAC layer design with directional antennas should start from another different perspective. For example, when the beamwidth is narrow enough and the collision probability is small, we can turn off RTS/CTS. On the contrary, we should consider other techniques, such as power control and multi-channel schemes to reduce interference.

\section{Conclusions}

This paper studies the performance of wireless networks using directional antennas with a narrow beam. In particular, we examine the probability of a successful transmission under Directional Transmission and Directional Reception. The numerical results show that the interference probability is quite low when the antenna beamwidth is narrow enough. These results encourage us to design a lightweight MAC protocol which turns off RTS/CTS. The evaluation results prove that the protocol has a higher throughput than the typical MAC protocol based on RTS/CTS. The results also demonstrate that a collision-tolerant transmission is feasible when the beamwidth is narrow enough. One of our future works is to implement the lightweight MAC protocol in simulators and conduct experiments in real environments.

\section{Acknowledgements}

This research was partially supported by Natural Science 
Foundation of China grant No.60573138 and 60773091, and the National Grand Fundamental Research 973 Program of China under Grant No.2006CB303000.

\section{References}

[1] Y. B. Ko, V. Shankarkumar, and N. H. Vaidya, "Medium access control protocols using directional antennas in ad hoc networks," in Proceedings IEEE INFOCOM, 2000.

[2] A. Nasipuri, S. Ye, and R. E. Hiromoto, "A MAC protocol for mobile ad hoc networks using directional antennas," in Proceedings IEEE WCNC, 2000.

[3] T. Korakis, G. Jakllari, and L. Tassiulas, "A MAC protocol for full exploitation of directional antennas in adhoc wireless networks," in Proceedings MobiHoc, 2003.

[4] M. Takai, J. Martin, R. Bagrodia, and A. Ren, "Directional virtual carrier sensing for directional antennas in mobile ad hoc networks," in Proceedings MobiHoc, 2002.

[5] R. R. Choudhury, X. Yang, N. H. Vaidya, and R. Ramanathan, "Using directional antennas for medium access control in ad hoc networks," in Proceedings MobiCom, 2002.

[6] C. S. Z. Huang, C.-C. Shen, and C. Jaikaeo, "A busytone based directional MAC protocol for ad hoc networks," in Proceedings MILCOM, 2002.

[7] R. R. Choudhury and N. H. Vaidya, "Deafness: a MAC problem in ad hoc networks when using directional antennas," in Proceedings ICNP, 2004.

[8] H. Singh and S. Singh, "Smart-802.11b MAC protocol for use with smart antennas," in Proceedings IEEE ICC, 2004.

[9] L. Bao and J. Garcia-Luna-Aceves, "Transmission scheduling in ad hoc networks with directional antennas," in Proceedings MobiCom, 2002.
[10] Z. Zhang, "Pure directional transmission and reception algorithms in wireless ad hoc networks with directional antennas," in Proceedings IEEE ICC, 2005.

[11] K. Xu, M. Gerla, and S. Bae, "How effective is the IEEE 802.11 RTS/CTS handshake in ad hoc networks," in Proceedings IEEE GLOBECOM, 2002.

[12] H. N. Dai, K. W. Ng, and M. Y. Wu, "A busy-tone based MAC scheme for wireless ad hoc networks using directional antennas," in Proceedings IEEE Globecom, 2007.

[13] IEEE 802.11, Part 11: Wireless LAN Medium Access Control (MAC) and Physical Layer (PHY) specifications - Amendment 4: Further Higher Data Rate Extension in the $2.4 \mathrm{GHz}$ Band, IEEE Std., 2003.

[14] A. Spyropoulos and C. S. Raghavendra, "Capacity bounds for ad-hoc networks using directional antennas," in Proceedings IEEE ICC, 2003.

[15] S. Yi, Y. Pei, and S. Kalyanaraman, "On the capacity improvement of ad hoc wireless networks using directional antennas," in Proceedings ACM MobiHoc, 2003.

[16] Y. Wang and J. J. Garcia-Luna-Aceves, "Directional collision avoidance in ad hoc networks," Performance Evaluation Journal, Vol. 58, pp. 215-241, 2004.

[17] M. M. Carvalho and J. J. Garcia-Luna-Aceves, "Modeling wireless ad hoc networks with directional antennas," in Proceedings IEEE INFOCOM, 2006.

[18] R. Ramanathan, "On the performance of ad hoc networks with beamforming antennas," in Proceedings Mobi-Hoc, 2001.

[19] T. S. Rappaport, "Wireless communications: principles and practice," 2nd edition Upper Saddle River, N.J.: Prentice Hall PTR, 2002.

[20] L. Wu and P. K. Varshney, "Performance analysis of CSMA and BTMA protocols in multihop networks (i). single channel case," Information Sciences, Vol. 120, pp. 159-177, 1999. 